\title{
Effects of oxygen and tetravinylsilane plasma treatments on mechanical and interfacial properties of flax yarns in thermoset matrix composites
}

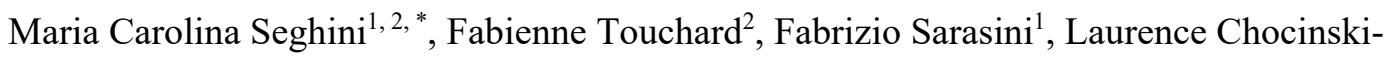
Arnault $^{2}$, Jacopo Tirillò ${ }^{1}$, Maria Paola Bracciale ${ }^{1}$, Milan Zvonek ${ }^{3}$, Vladimir Cech $^{3}$

${ }^{I}$ Department of Chemical Engineering Materials Environment and UdR INSTM, SapienzaUniversità di Roma, Via Eudossiana 18, 00184 Rome, Italy

${ }^{2}$ Institut PPRIME, CNRS-ENSMA-Université de Poitiers, Département Physique et Mécanique des Matériaux, ENSMA, 1, Av. Clément Ader, B.P. 40109, 86961 Futuroscope Cedex, France

${ }^{3}$ Institute of Materials Chemistry, Faculty of Chemistry, Brno University of Technology, Purkynova 118, CZ-612 00 Brno, Czech Republic

*Corresponding author: M.C. Seghini, mariacarolina.seghini@uniromal.it, mariacarolina.seghini@ensma.fr

\section{Abstract}

This work is focused on the assessment of the effect of oxygen and polymer plasma tetravinylsilane (pp-TVS) treatments on the adhesion of flax yarns with epoxy and vinylester thermoset matrices. These low temperature plasma processes have been selected as more environmentally friendly alternatives to traditional chemical treatments. Tensile tests performed on single flax yarns revealed a reduction in their mechanical properties after plasma treatments. In particular, a tensile strength reduction of $36.4 \%$ was detected after the oxygen plasma treatment using $100 \mathrm{~W}$ of plasma power. The morphological analysis highlighted that this result is mainly ascribed to the ablation action produced by oxygen plasma process. In the case of pp-TVS, both morphological and Fourier transform infrared spectroscopy analysis (FT-IR) confirmed the presence of a homogeneous tetravinylsilane film on the surface of the yarns. The interfacial adhesion of untreated, oxygen plasma treated, and plasma-polymer coated flax yarns has been determined by single fibre fragmentation test (SFFT). The plasma polymer deposition can produce a significant improvement of the adhesion property of flax yarns with both epoxy and vinylester matrices. An increase of the interfacial shear strength (IFSS) values of $114 \%$ and $71 \%$ was found after the TVS film deposition in epoxy and vinylester composites, respectively. These results were confirmed by high-resolution micro-CT, photoelasticity analysis and FE-SEM observations.

Keywords: Plant fibres, Interface/interphase, Fragmentation, Plasma treatment, micro-CT

\section{Introduction}

In the last two decades, the development of natural fibre reinforced polymer matrix composites has attracted great interest due to their properties and environmentally friendly character, with their global market that is forecast to grow at a CAGR (Compound Annual Growth Rate) of $8.2 \%$ from 2015 to 2020, especially driven by automotive, building and construction applications (Lucintel, 
2015). In particular, lignocellulosic fibres such as flax, hemp, jute, sisal and abaca have been proposed as substitutes of traditional glass fibres (Koronis, Silva, and Fontul, 2013)(Kiruthika, 2017). This growing attention towards the application of lignocellulosic fibres as reinforcement in polymers is not surprising as lignocellulosic fibres offer several advantages over conventional synthetic ones, such as low density, low cost, good specific mechanical properties, wide availability and biodegradability. Despite these benefits, the poor compatibility with polymer matrices still represents a major limitation for the industrial exploitation of this kind of fibres. In fact, their peculiar chemical composition, hydrophilic properties and surface characteristics prevent them from achieving a proper interfacial adhesion with hydrophobic polymer matrices, which results in composites with poor mechanical properties. To this purpose, various surface treatments have been proposed over the years, which have been the subject of extensive and detailed reviews (George et al., 2001; John and Anandjiwala, 2008; Kalia, Kaith, and Kaur, 2009; Li, Tabil, and Panigrahi, 2007; Mohanty, Misra, and Drazal, 2001). These treatments can be divided in physical (stretching, ultrasound, thermal and non-thermal plasma, electric discharge method, etc.) (De Almeida Mesquita et al. 2017; Ke et al. 2007; Molina 2016; Ragoubi et al. 2012; Yachmenev, Blanchard, and Lambert 1998) and chemical (alkalisation, acetylation, benzoylation, acrylation, bleaching, coupling agents etc.) methods (Amiandamhen, Meincken, and Tyhoda, 2018; Bulut and Aksit, 2013; Fiore, Scalici, and Valenza, 2017; Liu et al., 2016, 2017; Mukhopadhyay and Fangueiro, 2009; Ng, Shahid, and Nordin, 2018; Shanmugasundaram, Rajendran, and Ramkumar, 2018; B. Wang et al., 2003). Many of these techniques induce modifications of the surface chemistry of the fibres, while other treatments consist of a selective removal of fibre components that do not contribute to the reinforcement of the composite but have a negative effect on the adhesion quality. However, a large amount of hazardous chemicals is involved in these treatments to increase the hydrophobicity of natural fibres. Because of the stricter environmental legislation, process effluents must be handled and disposed of appropriately and, at this point, they represent a major cost contributor (Baltazar-y-Jimenez et al., 2008). For this reason, there is a need to develop more environmentally safe and effluent-free processes able to promote the adhesion between natural fibres and the polymeric matrices. In this framework, the plasma treatment represents a clean and environmentally friendly superficial modification technique. More specifically, plasma treatment does not employ harmful chemicals or gases, it leaves limited or no waste and it is able to modify only the surface of the fibre without affecting its internal properties. In addition, a limited amount of input precursor is needed, thus being an ultimately green process (Yasuda and Matsuzawa, 2005). In literature several studies have investigated the use of plasma treatment on natural fibres to improve the fibre/matrix adhesion. Scalici et al. (Scalici, Fiore, and Valenza, 2016) studied the effects of plasma treatment on the properties of Arundo Donax L. leaf fibres. From the remarkable enhancements of mechanical properties of the resulting composites, this study concluded that the plasma treatment can enhance the fibre/matrix adhesion. Low pressure plasma has been used by de Farias et al. (De Farias et al., 2017) to modify coir fibres. An increase in the surface roughness of fibres and an exposition of the crystalline cellulose were the main outcomes using air and oxygen gases, which resulted in improved mechanical properties of the composites. Baltazar-y-Jimenez et al. (Baltazar-y-Jimenez et al., 2008) reported that the atmospheric air pressure plasma treatment produced an increase in the interfacial shear strength values for flax, sisal and hemp fibres with cellulose acetate butyrate matrix, despite a decrease in mechanical properties of the fibres with increasing treatment duration. This increase was ascribed to the introduction of functional groups, the cleaning of contaminant substances and the enhanced surface roughness able to promote the mechanical interlocking between the fibre and the matrix. The effects of argon and air atmospheric pressure plasma on woven flax fibres have been explored by Bozaci et al. (Bozaci et al., 2013). The study showed that argon treatment is effective in increasing the roughness and so the adhesion of flax fibres with high density polyethylene (HDPE) matrix by mechanical interlocking. On the contrary, air plasma was found to strongly affect the oxygen/carbon ratio on the fibre surface thus promoting the adhesion with unsaturated polyester matrices. Most of the available studies in literature on surface modification of natural fibres used plasma treatment for surface etching and/or 98 functionalization. In this context, the aim of the present work is to investigate the effects of two 
different plasma treatments on the adhesion quality of flax yarns to different thermoset resins. In particular, an oxygen plasma and a plasma polymerization treatment have been performed on flax yarns. The oxygen plasma has been selected as it is able to increase the surface roughness and introduce functional groups such as $-\mathrm{OH}, \mathrm{C}-\mathrm{O}, \mathrm{C}=\mathrm{O}$ and $\mathrm{O}-\mathrm{C}=\mathrm{O}$ in the surface of flax fibres, thus resulting in improved wettability. The plasma polymerization treatment consists in a plasma polymerized film deposition (Plasma Enhanced Chemical Vapor Deposition - PECVD) that can increase the wettability and consequently the fibre/matrix adhesion through an engineered deposition of a compatible interlayer on the fibre surface (Cech et al., 2014). Monomer and gas molecules are fragmented and ionized in plasma producing excited species, free radicals and ions. Adsorbed activated fragments recombine forming a thin plasma polymer film onto the fibre surface. In this study a tetravinylsilane monomer has been selected to produce a thin plasma polymer tetravinylsilane film (pp-TVS). Unlike the most common coating processes, such as silanization or polymer grafting, through the plasma polymerization process it is possible to obtain very thin films reducing the amount of chemical species used. Another positive feature of the selected plasma polymerization process is its suitability for industrial scale-up, as recently demonstrated on glass fibres ( Cech et al., 2019).

In this paper, the impact of plasma treatment on the mechanical properties of flax yarns has been determined by performing single yarn tensile tests. The influence of plasma on the functional groups and the analysis of the thermal stability of untreated and plasma treated flax yarns have been assessed by Fourier transform infrared spectroscopy (FTIR) and thermogravimetric (TGA) analysis, respectively. In this study the single fibre fragmentation test (SFFT) has been transferred to the scale of single flax yarn specimens to investigate the effect of plasma treatment on the adhesion quality of flax with two different types of thermoset matrices, namely epoxy and vinylester (Seghini et al., 2018). The quality of the interfacial adhesion between flax yarns and both matrices has been assessed in terms of critical fragment length, debonding length and interfacial shear strength (IFSS). High-resolution microtomography $(1.5 \mu \mathrm{m})$, photoelasticity and FE-SEM observations allowed a better understanding of the interfacial adhesion phenomena.

\section{Materials and methods}

\subsection{Materials}

Individual flax yarns were carefully extracted by hand from the Biotex flax fabric supplied by Composites Evolution, which is an untreated $2 \times 2$ twill fabric with an areal weight of $200 \mathrm{~g} / \mathrm{m}^{2}$. The epoxy matrix consisted of the Epoxy Prime 27 infusion resin and PRIME 20 slow hardener by GURIT. The curing process was carried out with a mixing ratio of 100:28 by weight. The Advalite VH-1207 styrene free vinylester resin, supplied by Reichhold, was selected as the second thermoset matrix. The Norpol Peroxide PMEC N24 catalyst, supplied by Reichhold, was used as curing agent (mixing ratio of $1 \mathrm{phr}$ ).

\subsection{Plasma Modification Treatments of Flax Surface}

Plasma surface treatments were carried out in a reactor consisting of a glass tube $100 \mathrm{~cm}$ long and with an inner diameter of $40 \mathrm{~mm}$. The plasma system was first evacuated to a basic pressure of about $5 \times 10^{-4} \mathrm{~Pa}$. Argon gas (99.999\%) was used to clean the plasma reactor and vacuum chambers. A first oxygen plasma treatment has been performed. Plasma was excited at powers of $2,10,20,50$ and $100 \mathrm{~W}$. The flax surfaces were exposed to a continuous oxygen plasma ablation for $30 \mathrm{~min}$ at $5.8 \mathrm{~Pa}$. Subsequently, a plasma polymerization treatment has been performed on flax yarns. The polymer deposition treatment has been divided in two steps: a first non-polymerising gas plasma step, a pre-treatment with oxygen plasma for $30 \mathrm{~min}$ to remove contaminants and 
improve film adhesion, and a second step of polymerising gas plasma, in which tetravinylsilane monomer $\mathrm{Si}\left(-\mathrm{CH}=\mathrm{CH}_{2}\right)_{4}$ (purity $97 \%$, Sigma Aldrich) was fragmented and ionized in plasma producing excited species, free radicals and ions which were recombined forming a thin plasma polymer film onto the yarns surface. Tetravinylsilane plasma was employed for $15 \mathrm{~min}$. In order to assess the effect of the oxygen pretreatment step on the deposition quality, oxygen plasma was excited using two different power conditions, $2 \mathrm{~W}$ and $100 \mathrm{~W}$, respectively at $5.8 \mathrm{~Pa}$. In both cases, the plasma deposition step was carried out using a power of $10 \mathrm{~W}$ at $3.8 \mathrm{~Pa}$.

\subsection{Optical and FE-SEM observations}

The flax yarn diameters and all the fragment lengths were measured by using a ZEISS Axio Imager optical microscope. A morphological investigation of the surface of plasma treated and untreated flax yarns has been carried out using a field-emission gun scanning electron microscope (FE-SEM) Zeiss Auriga. After single fibre fragmentation tests, the morphology of the single yarn composite fracture surfaces was investigated by a FE-SEM JEOL JSM-7000F. All specimens were sputter coated with chromium prior to FE-SEM observation.

\subsection{Tensile testing}

Tensile properties of untreated and plasma treated flax yarns have been determined by single yarn tensile tests in accordance with ASTM C-1557 (Seghini et al., 2018). Individual yarns were glued onto card tabs with a central window cut out to match the desired gauge length for the test. For each family, at least thirty tests have been performed using a gauge length of $40 \mathrm{~mm}$. Tensile tests were carried out at room temperature with a Zwick/Roell Z010 equipped with a $1 \mathrm{kN}$ load cell. Tests were performed in displacement control with a cross-head speed of $2 \mathrm{~mm} / \mathrm{min}$. Before testing, all the yarns were conditioned at $45^{\circ} \mathrm{C}$ for $24 \mathrm{~h}$ for moisture elimination.

\subsection{Fourier transform infrared spectroscopy analysis (FT-IR)}

The chemical composition of the as-received and plasma treated flax fibres has been studied by Fourier-transform infrared (FTIR) analysis. Infrared measurements were carried out with a Bruker Vertex 70 spectrometer (Bruker Optik $\mathrm{GmbH}$ ) equipped with a single reflection Diamond ATR cell. Spectra were recorded with a $3 \mathrm{~cm}^{-1}$ spectral resolution in the mid infrared range (400-4000 $\mathrm{cm}^{-1}$ ) using 512 scans.

\subsection{Thermogravimetric analysis (TGA)}

Analysis of the mass loss of untreated and plasma treated flax yarns with temperature has been carried out using a SetSys Evolution (Setaram Instrumentation) thermogravimetric analyser. The different yarns were placed in an alumina pan and heated at a rate of $10^{\circ} \mathrm{C} / \mathrm{min}$ to a maximum temperature of $800{ }^{\circ} \mathrm{C}$ in nitrogen atmosphere.

\subsection{Photoelasticity analysis}

To study the distribution of interfacial stresses in the single yarn reinforced composites, a photoelasticity analysis has been performed for the untreated flax yarn/epoxy and the tetravinylsilane plasma treated flax yarn/epoxy systems. During single fibre fragmentation tests, specimens were placed into a circular polariscope in order to reveal the stress state near yarn breaks and at the interface between the yarn and the matrix by photoelasticity. An AVT-Dolphin high resolution camera, focusing on the reduced gauge section of the specimens, has been used during tests. To ensure a continuous recording, pictures have been taken every $2 \mathrm{~s}$. 
The single fibre fragmentation test has been used in a previous experimental work to assess the interfacial properties of untreated flax yarns with epoxy and vinylester matrices (Seghini et al., 2018). A metallic mould has been used for the manufacture of composite specimens reinforced with a single flax yarn aligned along the load direction. Each flax yarn was positioned in the mould and held in place by adhesive tape. Before casting, flax yarns were conditioned at $45^{\circ} \mathrm{C}$ for $24 \mathrm{~h}$ for moisture elimination. A dog bone specimen, characterised by a reduced gage section with a length of $15 \mathrm{~mm}$, a thickness of $2 \mathrm{~mm}$ and a width of $3 \mathrm{~mm}$, has been produced. The fragmentation testing was performed with an Instron E1000 ElectroPuls test machine with a load cell of $2 \mathrm{kN}$ and using a crosshead speed of $0.005 \mathrm{~mm} / \mathrm{min}$. For each specimen, the yarn diameter has been measured in at least ten points along the gauge length by optical microscopy before each test. During tests, the loading phase was stopped if the specimen failed, or when the fragmentation saturation level was achieved, which was defined as the point when no new yarn breaks appeared during a subsequent strain increase by $0.5 \%$. In order to be sure to reach the saturation level, epoxy- and vinylester-based specimens were loaded up to failure at strains higher than 9 and $11 \%$, respectively.

\subsection{Micro-CT apparatus}

An UltraTom CT scanner manufactured by RX Solutions (France) has been used for the image acquisition. High resolution micro-CT observations have been performed for both epoxy and vinylester composites with untreated and PECVD flax yarns. A resolution of $1.5 \mu \mathrm{m}$ has been used in this work, with an accelerating voltage of $50 \mathrm{kV}$ and a beam current of $157 \mu \mathrm{A}$. A volumetric reconstruction has been performed for both epoxy and vinylester systems with the untreated and the $100 \mathrm{~W}$ oxygen-PECVD treated flax yarns. For 3D reconstruction, images were acquired from 1120 rotation views over $360^{\circ}$ of rotation ( 0.32 rotation step). The analysis of the micro-CT pictures has been performed by the Avizo 9.0 software.

\section{3- Results and discussion}

\subsection{Characterization of flax yarns}

A morphological analysis was carried out on the surface of flax yarns treated by oxygen plasma. Figure 1 shows the SEM micrographs of the lateral surface of untreated flax yarns and oxygen plasma treated flax yarns using the power of 2,10,20,50 and $100 \mathrm{~W}$. As the plasma power increases, an enhancement in the surface roughness of the flax yarns can be observed, which is essentially linked to the ability of the reactive plasma gas to perform a surface ablation of the flax fibres. Ablation occurs by a chemical etching process during the plasma treatment. In fact, the oxygen plasma is a chemically reactive plasma in which the inorganic oxygen gas is not able to form any polymeric deposition. The ionization and dissociation reactions of oxygen gas are

The reaction of these high energy oxygen ions with the flax surface is able to produce changes in the surface morphology. As reported by Sun and Stylios (Sun and Stylios, 2006), after the oxygen 

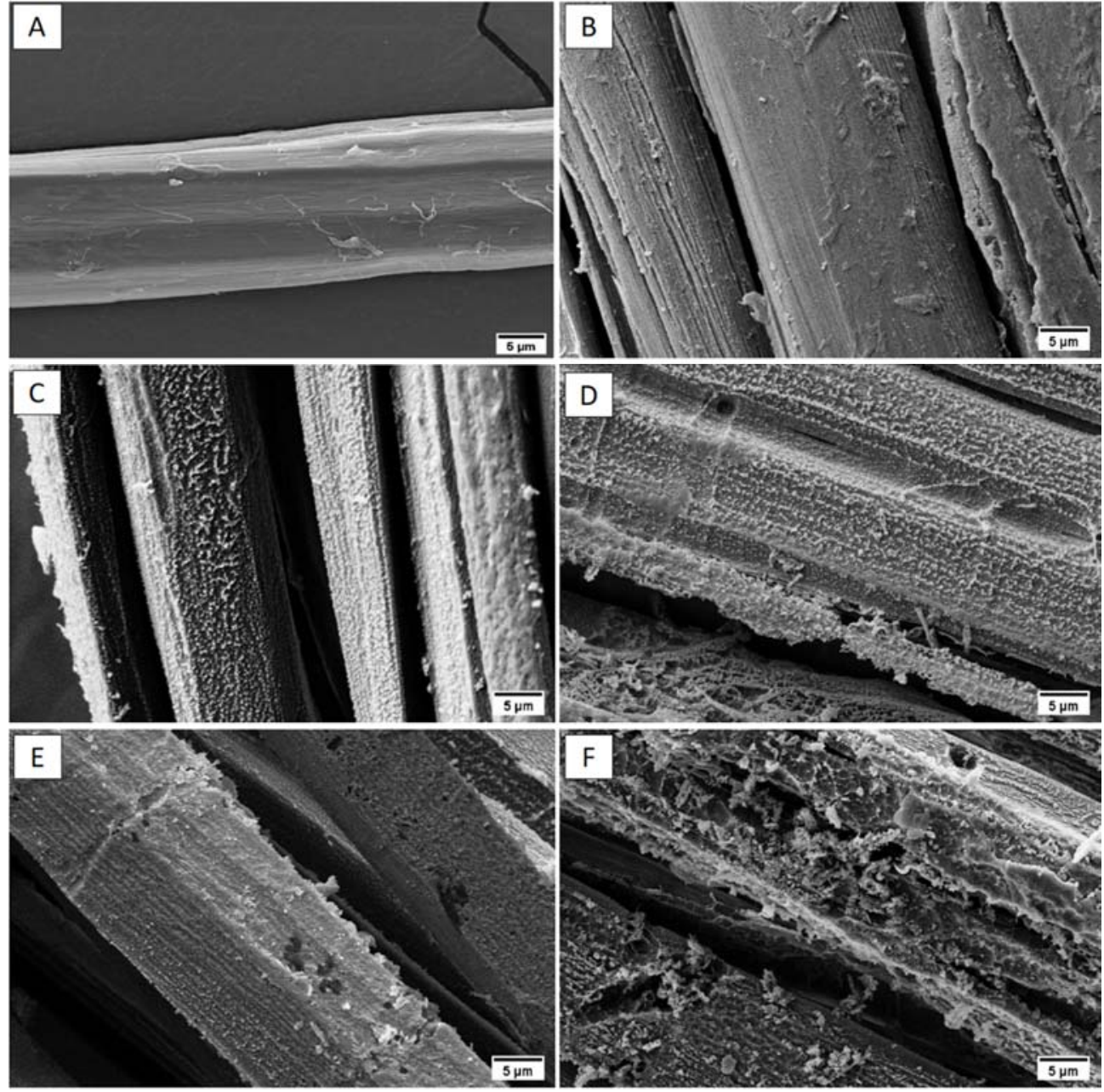

Figure 1 SEM micrographs detailing the lateral surface of untreated flax yarn (A) and oxygen plasma treated flax yarn using a power of $2 \mathrm{~W}(\mathrm{~B}), 10 \mathrm{~W}(\mathrm{C}), 20 \mathrm{~W}(\mathrm{D}), 50 \mathrm{~W}(\mathrm{E})$ and $100 \mathrm{~W}(\mathrm{~F})$.

Similar results were found by De Farias et al. (De Farias et al., 2017). This study showed that the oxygen plasma treatment of coir fibres led to a partial removal of the surface amorphous layer, revealing the inner structure with long valleys and peaks. The same morphological analysis has been carried out after the plasma deposition of the polymeric TVS film. This analysis showed that the quality of the polymeric coating strictly depends on the plasma power used during the first oxygen non-polymerising gas plasma step. Oxygen power values of $100 \mathrm{~W}$ and $2 \mathrm{~W}$ have been tested. By comparing the SEM micrographs reported in Figures 2-A, 2-B and Figures 3-A, 3-B, it can be seen that the oxygen pre-treated flax yarns with a plasma power of $2 \mathrm{~W}$ are characterized by the presence of a very homogeneous polymeric layer. A much more uneven coating was obtained for the flax yarns treated using a power of $100 \mathrm{~W}$. The morphological analysis confirms that the final characteristics of the polymer film strongly depend on the finishing of lateral surface of flax yarn as a result of the oxygen pre-treatment. The higher the plasma oxygen power used, the greater the roughness of the flax fibres and so the lower the homogeneity of the polymeric film. 

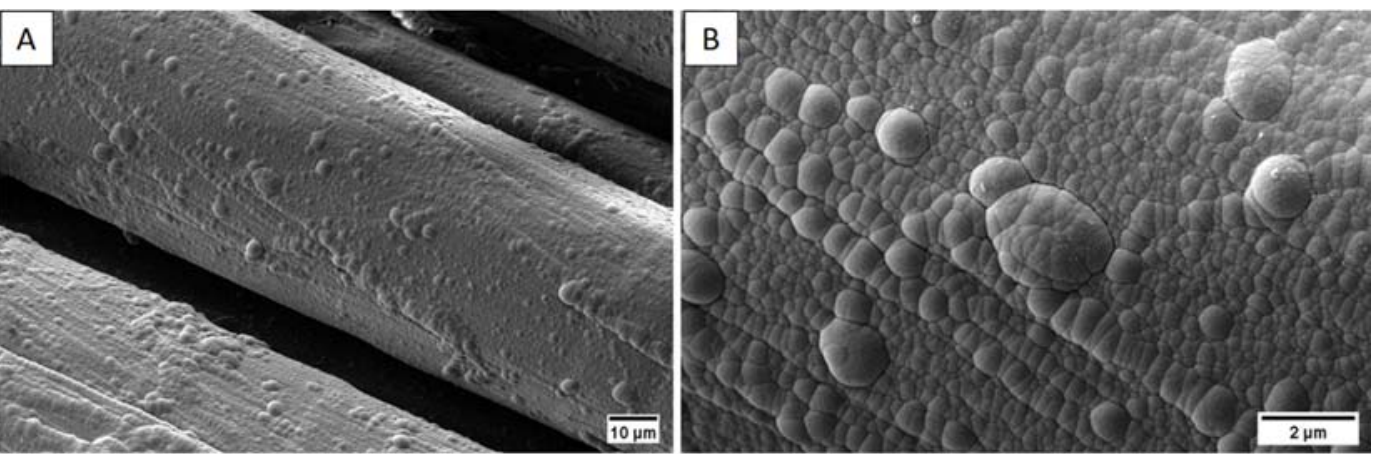

Figure 2 SEM micrographs of flax yarns after the plasma polymer tetravinylsilane deposition, using 270 a plasma power of $2 \mathrm{~W}$ for the oxygen pre-treatment. Lateral surface of treated flax fibres (A) and a detailed view of the polymer film (B).
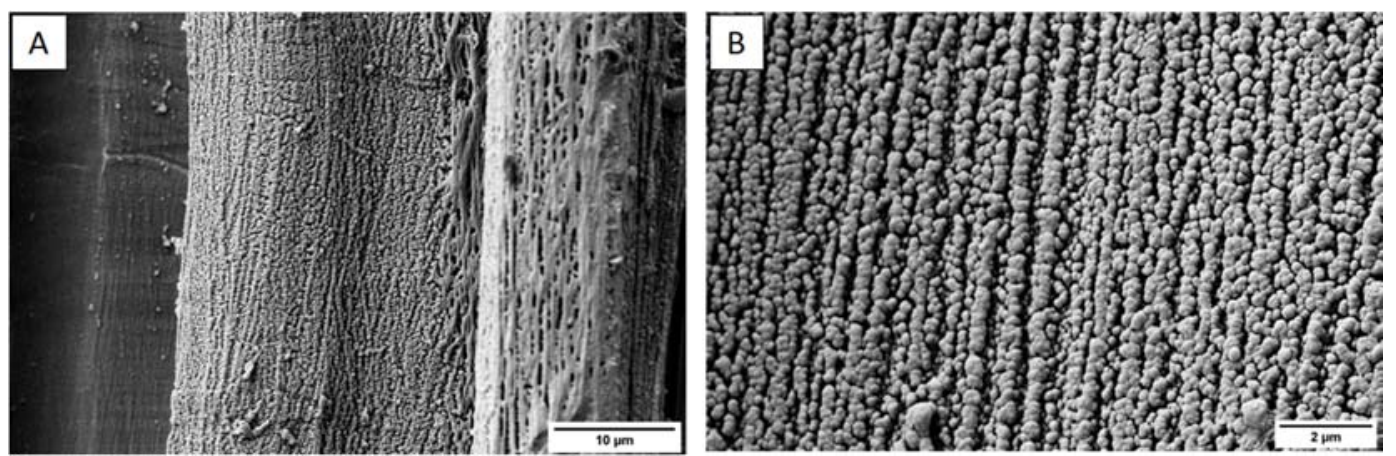

Figure 3 SEM micrographs of flax yarns after the plasma polymer tetravinylsilane deposition using a plasma power of $100 \mathrm{~W}$ for the oxygen pre-treatment. Lateral surface of treated flax fibres (A) and a detailed view of the polymer film (B).

The effect of the different plasma treatments on the chemical composition of flax fibres has been studied by Fourier-transform infrared (FTIR) analysis. FT-IR spectra in Figure 4 show the untreated and the oxygen plasma treated flax fibres using $2 \mathrm{~W}$ and $100 \mathrm{~W}$ power values. It can be observed that untreated flax fibre consists of alkene, esters, aromatics, ketone and alcohol belonging to cellulose, hemicellulose and lignin (Bozaci et al., 2013; Reddy et al., 2015; Titok et al., 2010), as reported in table 1 .

Table 1 Assignment of the main ATR-FTIR bands.

Wavenumber $\left(\mathrm{cm}^{-1}\right)$

3700-3000

2990-2754

1734

1629

$1597,1511,1450$

$1426,1365,1314$

1278

$1251,1159,1105,1028$

1053

897

\section{Band Assignments*}

$\nu \mathrm{OH}$

$v \mathrm{CH}$ of cellulose and hemicellulose

$$
\nu \mathrm{C}=\mathrm{O} \text { of hemicellulose }
$$

$\delta \mathrm{HOH}$ of water in crystalline cellulose

$v \mathrm{C}=\mathrm{C}$ and $d \mathrm{CH}$ in methyl, methylene and methoxyl groups of lignin $\delta \mathrm{CH}_{2}, \delta \mathrm{CH}$ of cellulose and $\omega \mathrm{CH}_{2}$ in cellulose and hemicellulose

$$
\tau \mathrm{C}-\mathrm{H}_{2} \text { of cellulose }
$$

$v \mathrm{C}$-O-C and $v \mathrm{C}$-C of polysaccharide components (mainly cellulose)

$v \mathrm{C}$-OH of cellulose and hemicellulose

$\beta$-glycosidic linkages between the sugars units in cellulose

* $v=$ stretching; $\delta=$ bending; $\mathrm{d}=$ deformation; $\omega=$ wagging; $\tau=$ twisting. 


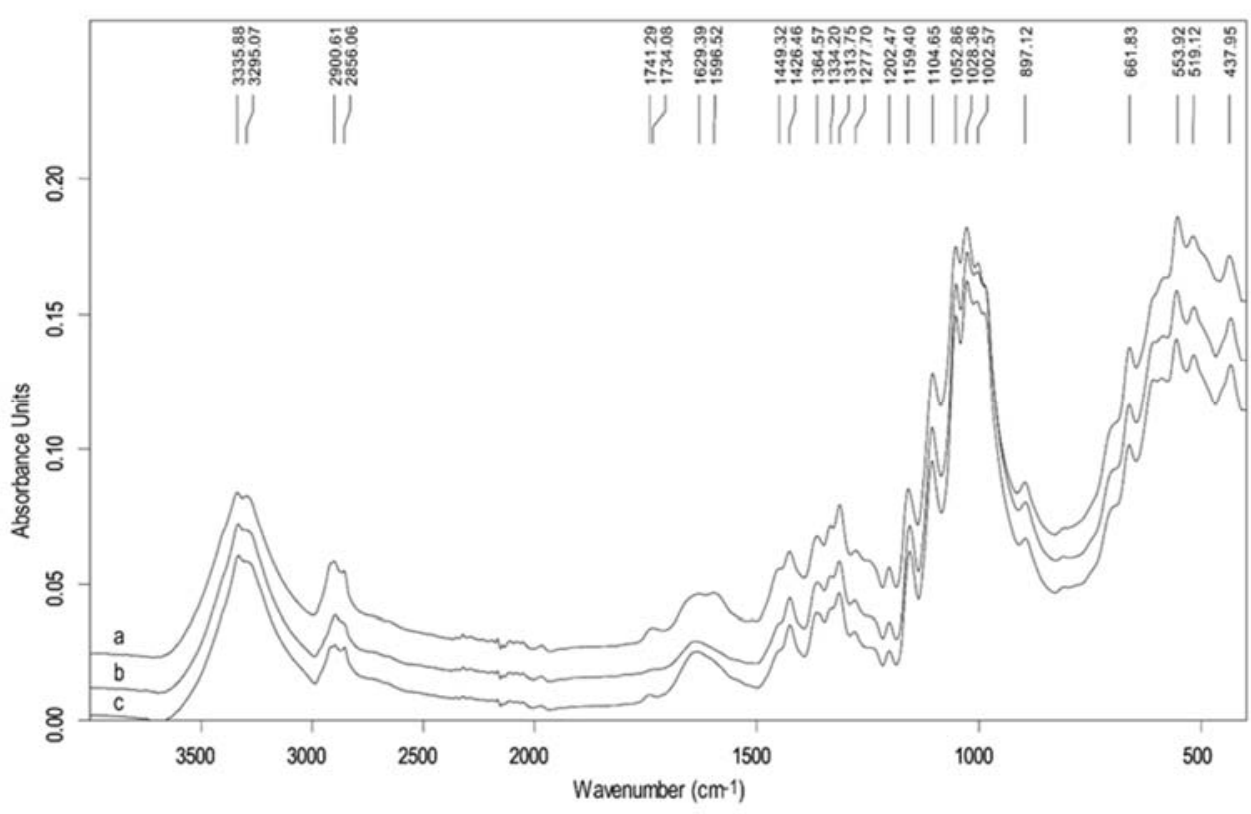

Figure 4 Infrared spectra of different flax yarns: untreated (a), O2-plasma treated at $2 \mathrm{~W}$ (b) and $100 \mathrm{~W}(\mathrm{c})$.

Upon plasma pre-treatment, the bands in the range $1780-1520 \mathrm{~cm}^{-1}$ changed, indicating that hemicelluloses and lignin in the fibres were degraded as shown by the decrease of the bands at 1734 and 1597, $1511 \mathrm{~cm}^{-1}$, respectively. This is in agreement with previous studies, which demonstrated that the amorphous regions are more susceptible to chemical and ion etching (Donaldson and Frankland 2004; Jamali and Evans 2011; De Souza Lima and Borsali 2004; Warner, Uhlmann, and Peebles Jr. 1975). In addition, with $\mathrm{O}_{2}$ pre-treatment at $100 \mathrm{~W}$, an increase at $\approx 1630 \mathrm{~cm}^{-1}$ can be attributed to the breakage of the $\beta-1,4$ glycosidic bonds of cellulose macromolecules in the oxidation reaction of the cellulose as confirmed by the reduction of the integrated intensity of the band at $897 \mathrm{~cm}^{-1}(2 \mathrm{~W}: 0.085 ; 100 \mathrm{~W}: 0.076)$. Indeed this latter, as previously reported, was found to be linearly correlated with the percentage of cellulose (Noureddine Abidi, Cabrales, and Haigler, 2014) and degree of crystallinity (N Abidi and Manike, 2017). Moreover, a new band at $1741 \mathrm{~cm}^{-1}$, probably due to the formation of oxidized species rich in hydroxyl, carbonyl, carboxyl groups and phenoxy radicals, appeared after $\mathrm{O}_{2}$ plasma treatment at 100W (Demirkir, Colak, and Ozturk, 2017). The presence of the polymeric layer has been confirmed also by infrared spectroscopy analysis. Typical infrared bands of plasma-polymerized tetravinylsilane (pp-TVS) film deposited on flax fibres are shown in Figure 5. In the wavenumber range 3700-3200 $\mathrm{cm}^{-1}$ a significant band of $-\mathrm{OH}$ and harmonic vibration of $\mathrm{C}=\mathrm{C}$ in vinyl group at $3286 \mathrm{~cm}^{-1}$ occurs. Following, wide bands in the range $3100-2750 \mathrm{~cm}^{-1}$ can be ascribed to vibrations of $-\mathrm{CH}_{2}$ groups. Significant bands are the ones related to $\mathrm{Si}-\mathrm{H}$ and $\mathrm{C}=\mathrm{O}$ vibration at $2112 \mathrm{~cm}^{-1}$ and $1696 \mathrm{~cm}^{-1}$, respectively. Four bands contribute to $\mathrm{Si}-\mathrm{CH}=\mathrm{CH}_{2}$ vibrations, such as: $\mathrm{CH}_{2}$ scissoring $\left(1451 \mathrm{~cm}^{-1}\right),=\mathrm{CH}_{2}$ deformation in vinyl group $\left(1404 \mathrm{~cm}^{-1}\right),-\mathrm{CH}_{2}$ wagging in Si$\mathrm{CH}_{2}-\mathrm{R}\left(1250 \mathrm{~cm}^{-1}\right)$ and $=\mathrm{CH}\left(1021 \mathrm{~cm}^{-1}\right), \mathrm{CH}_{2}$ wagging (shoulder at $\left.950 \mathrm{~cm}^{-1}\right)$ of $=\mathrm{CH}_{2}$ bond. Finally, a multiband in the range $1100-1000 \mathrm{~cm}^{-1}$ belongs to Si-O-Si stretching $\left(1097 \mathrm{~cm}^{-1}\right)$, Si-O$\mathrm{C}$ stretching $\left(1050 \mathrm{~cm}^{-1}\right)$ and Si-O $\left(869 \mathrm{~cm}^{-1}\right)$ bending (V Cech et al., 2007, 2014; Davidson, 1971). 


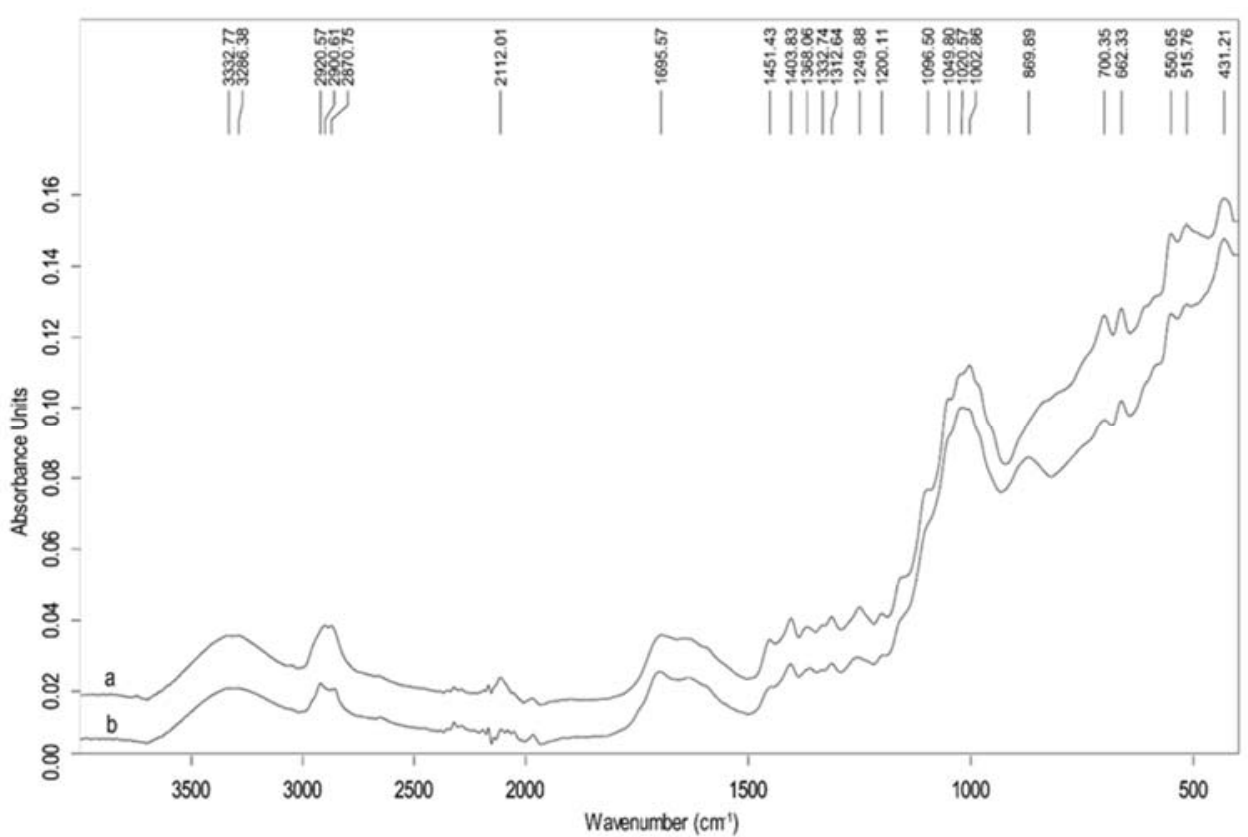

Figure 5 Infrared spectra of pp-TVS films deposited on pre-treated flax yarns at 2W (a) and 100W (b).

Thermogravimetric analysis has been used to assess the thermal stability of untreated and both oxygen and tetravinylsilane plasma treated flax yarns. Weight changes versus temperature and the derivative of weight changes versus temperature have been measured (Figure 6). Thermographs reveal three peaks in the derivative curves for the different flax fibres: the first mass loss, at about $60-160^{\circ} \mathrm{C}$, is related to the release of water, the second peak, at about $240-280^{\circ} \mathrm{C}$, is attributed to the decomposition of the non-cellulosic components such as pectin and hemicellulose and the third mass loss peak, at about $340-360^{\circ} \mathrm{C}$, is due to the cellulose degradation (Mazian et al., 2018). From the results reported in table 2, it can be noticed that the flax fibres treated using an oxygen plasma power of $2 \mathrm{~W}$ show similar behaviour to the untreated one. A strong influence on thermal stability of flax can be instead detected starting from oxygen plasma power of $10 \mathrm{~W}$. De Oliveira et al. (De Oliveira et al., 2017) highlighted as the oxygen plasma exposure can be able to perform a partial removal of non-cellulosic substances like hemicellulose, lignin, pectin, waxes and impurities from coconut surfaces. Different studies in the literature reported as removal of noncellulosic components from the fibres surface through chemical treatments can produce an increase in their thermal stability (Dong et al., 2015; Mazian et al., 2018; Velde and Baetens, 2001). The decrease in both decomposition peak temperature and $10 \%$ weight loss temperature of flax after the oxygen plasma exposure may be explained in an excessive value of plasma power used. In fact, the surface etching produced by oxygen can generate an overall degradation of flax fibres, with a consequent reduction in their thermal stability. By comparing the results found for the neat and the treated flax yarns using an oxygen plasma power of $100 \mathrm{~W}$, a $10{ }^{\circ} \mathrm{C}$ reduction can 


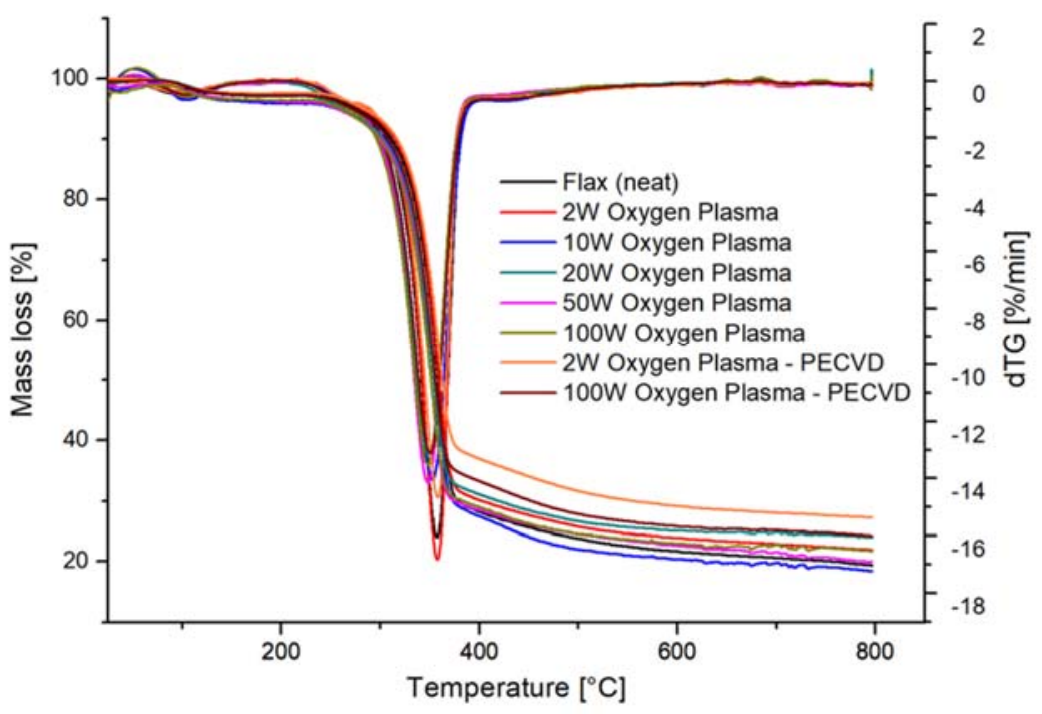

Figure 6 Mass loss and derivative of the mass loss as a function of temperature for the untreated and plasma treated flax yarns.

A positive effect on the thermal stability of the flax fibres has been observed after the pp-TVS deposition. Similar decomposition peak temperature values may be found for the $2 \mathrm{~W}$ and the $2 \mathrm{~W}$ PECVD treated flax yarns, whereas an increase has been measured for the 100W-PECVD treated yarns with respect to the $100 \mathrm{~W}$ oxygen treated ones.

Table 2 Results of the thermogravimetric analysis for the different kinds of flax yarns.

\begin{tabular}{ccc}
\hline \hline Flax yarn (neat) & $\begin{array}{c}\text { Peak maximum } \\
\text { Temperature }\left[{ }^{\circ} \mathbf{C}\right]\end{array}$ & $\begin{array}{c}\text { Temperature of 10 \% weight } \\
\text { loss }\left[{ }^{\circ} \mathbf{C}\right]\end{array}$ \\
Flax yarn 2W Oxygen Plasma & 357.2 & 311.3 \\
Flax yarn 10W Oxygen Plasma & 357.7 & 314.7 \\
Flax yarn 20W Oxygen Plasma & 352.5 & 304.3 \\
Flax yarn 50W Oxygen Plasma & 349.6 & 303.4 \\
Flax yarn 100W Oxygen Plasma & 348.0 & 301.0 \\
Flax yarn 2W Oxygen Plasma- PECVD & 347.9 & 299.7 \\
Flax yarn 100W Oxygen Plasma- PECVD & 357.9 & 316.9 \\
\hline \hline
\end{tabular}

In order to evaluate the effects of the different surface modification treatments on the mechanical performance of the single flax yarns, a mechanical characterization has been carried out by single yarn tensile tests. A brittle behaviour under tensile loading has been found for all the different families of flax yarns. From the results reported in table 3 , it is possible to notice a decrease in the maximum force and strength values with increasing power of oxygen plasma. 
Table 3 Tensile properties of flax yarns after different oxygen plasma power conditions and plasma deposition processes. In the table are also reported the parameters of the Weibull distribution for the tensile strength.

\begin{tabular}{|c|c|c|c|c|c|c|}
\hline & $\mathbf{F}_{\max }[\mathbf{N}]$ & $\begin{array}{c}\text { Diameter } \\
{[\mu \mathrm{m}]}\end{array}$ & $\sigma_{\mathrm{f}}[\mathrm{MPa}]$ & $\varepsilon_{\max }[\%]$ & $\begin{array}{c}\text { Characteristic } \\
\text { strength } \sigma_{0} \\
\text { [MPa] }\end{array}$ & $\begin{array}{c}\text { Weibull } \\
\text { Modulus } \\
\text { m }\end{array}$ \\
\hline Flax yarn neat & $19.8 \pm 4.8$ & $327 \pm 95$ & $236 \pm 57$ & $3.4 \pm 0.42$ & 257.4 & 5 \\
\hline Flax yarn $2 W$ Oxygen Plasma & $20.9 \pm 2.8$ & $319 \pm 60$ & $262 \pm 35$ & $3.67 \pm 0.33$ & 277 & 8.8 \\
\hline Flax yarn 10W Oxygen Plasma & $17 \pm 3.4$ & $330 \pm 85$ & $199 \pm 39$ & $3.39 \pm 0.33$ & 215 & 6.1 \\
\hline Flax yarn 20W Oxygen Plasma & $17.9 \pm 4.5$ & $314 \pm 90$ & $231 \pm 58$ & $3.49 \pm 0.44$ & 253.6 & 4.5 \\
\hline Flax yarn 50W Oxygen Plasma & $10.6 \pm 4$ & $307 \pm 97$ & $143 \pm 54$ & $2.71 \pm 0.5$ & 160 & 3.1 \\
\hline Flax yarn 100W Oxygen Plasma & $9.2 \pm 2.9$ & $280 \pm 69$ & $150 \pm 48$ & $2.62 \pm 0.56$ & 167.1 & 3.4 \\
\hline Flax yarn PECVD $\left(2 \mathrm{WO}_{2}\right)$ & $21 \pm 3.9$ & $355 \pm 87$ & $212 \pm 39$ & $2.96 \pm 0.35$ & 228.8 & 6 \\
\hline Flax yarn PECVD $\left(100 \mathrm{~W} \mathrm{O}_{2}\right)$ & $14.7 \pm 4.1$ & $277 \pm 63$ & $244 \pm 68$ & $2.81 \pm 0.59$ & 272.5 & 3.6 \\
\hline
\end{tabular}

For the $2 \mathrm{~W}$ oxygen plasma, the measured strength value can be considered similar to the one obtained for untreated flax yarns because of the large scattering. Then, for higher power, the strength values decrease, until a reduction of $36.4 \%$ for the $100 \mathrm{~W}$ condition. This result can be explained by the outcomes of the morphological analysis (Figure 1): the oxygen plasma carries out a surface ablation of flax fibres, generating defects such as pits, voids and spaces. Moreover, as shown in the thermogravimetric analysis, exposure of flax fibres to increasing plasma powers leads to a removal of hemicellulose, pectin and waxes but also to a strong degradation of structural components such as lignin and cellulose. The mechanical performance of flax yarns is thus closely linked to the oxygen plasma treatment. Concerning the results obtained for flax yarns treated with the TVS plasma, the average strength value measured for the yarns pretreated at $2 \mathrm{~W}$ is very similar to the one obtained for the yarns only treated with the $2 \mathrm{~W}$ power oxygen plasma. On the contrary, significantly higher strength values have been found for the $100 \mathrm{~W}$ pretreated flax $(244 \mathrm{MPa} \pm 68$ $\mathrm{MPa}$ ) than the yarns treated only with the $100 \mathrm{~W}$ power oxygen plasma $(150 \mathrm{MPa} \pm 48 \mathrm{MPa})$. This enhancement of the mechanical properties of the TVS plasma treated flax yarn may be related to the filling and healing process of superficial defects by the polymeric layer deposited on flax yarns. The experimental results were statistically analysed using a two-parameter Weibull distribution, according to the equation 3 (Sarasini, Tirillò, and Seghini, 2018):

$$
\operatorname{Pr}(\sigma)=1-\exp \left[-\left(\frac{\sigma}{\sigma_{0}}\right)^{m}\right]
$$

where $\operatorname{Pr}(\sigma)$ is the probability of survival of the tensile strength, $m$ is the Weibull modulus (related to the dispersion of the data) and $\sigma_{0}$ is the characteristic strength. Equation 4 gives the estimator, $\mathrm{P}_{\mathrm{f}}$, used for the probability of failure evaluation:

$P_{f}=\frac{i-o .5}{N}$

where $N$ is the number of filaments tested and $i$ is the rank of data point for each filament. In table 3 are reported all the Weibull moduli and the characteristic strength values found for the treated and untreated flax yarns. The curves reported in Figure 7 represent the results of the two-parameter Weibull distribution applied to all the analysed flax yarns. The data appear to be well-fitted by a two-parameter Weibull distribution. 


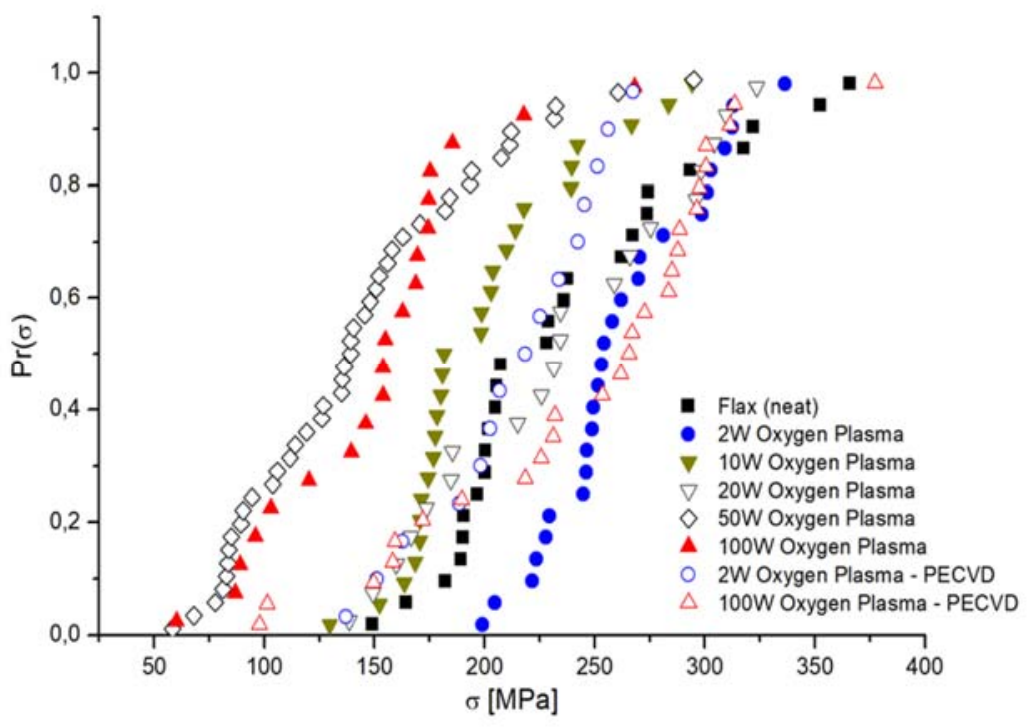

398 Figure 7 Cumulative distributions of the tensile failure probability values measured for the different 399 families of flax yarns.

\section{2 - Determination of critical fragment length and debonding length}

Fragmentation tests have been performed for the untreated flax yarns and for the flax yarns treated using oxygen and tetravinylsilane plasma with both epoxy and vinylester resins. In order to assess the effect of the plasma process on the adhesion quality of flax yarn, fragmentation tests have been performed only on yarns treated with the maximum and minimum oxygen plasma power values, $100 \mathrm{~W}$ and $2 \mathrm{~W}$, respectively. The critical fragment length value reflects the stress transfer efficiency between the fibre and the matrix at the interface. For this reason, the critical fragment length estimation plays a crucial role in the assessment of the adhesion quality in a composite material. As reported by Ohsawa et al. (Ohsawa et al., 1978), the critical fragment length may be evaluated using equation 5 :

where $\overline{1}$ is the average value of the fragment length. In figure 8 are reported examples of optical micrographs obtained for a fragmented flax yarn. For each sample, the yarn diameter and fragment lengths have been measured in the gauge length zone. As it is possible to see in Figure 8-A, the fragment lengths have been determined as the average values between the internal and the central points of the breaking zones. As it has been demonstrated in a previous experimental work through the micro-CT analysis (Seghini et al., 2018), the black area around each yarn break corresponds to the debonding length ( $l_{\text {debonding }}$ ) between flax yarn and the polymer resin. These lengths have been measured by optical microscopy (Figure 8-B) and all the values found for the different systems are 
A

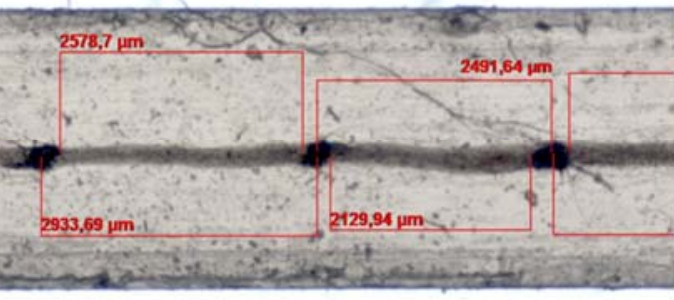

$\overline{500 \mu \mathrm{m}}$
424

425

426

427

428

429

430

431

432

433

434

435

436

437

438

439

440

Figure 8 Typical optical micrographs of a fragmented flax yarn: measurement of the yarn fragment length 1 (A) and of the debonding length ldebonding (B).

When comparing the results found for the untreated flax yarns with those for the oxygen plasma treated yarns in epoxy resin systems, only the $100 \mathrm{~W}$ process is able to reduce the critical and debonding length values. The oxygen plasma treatment performed at a power of $2 \mathrm{~W}$ seems to be able to decrease the length of the debonding zone between the flax yarn and the epoxy matrix at the expense of an increased critical length. These results could lead to the conclusion that only an oxygen plasma treatment with a power of $100 \mathrm{~W}$ is able to effectively modify the surface of flax yarns and increase the adhesion with the epoxy matrix. For the flax yarn/vinylester systems, it can be seen that debonding and critical length values are significantly higher than those obtained for the epoxy resin. These results show that the flax yarn/epoxy system is characterized by a better interface quality than the flax/vinylester one.

Table 4 Critical fragment length and debonding length values for the different single yarn composites.

\begin{tabular}{|c|c|c|c|}
\hline Matrix & Flax yarn & $1_{\text {debonding }}[\mu \mathrm{m}]$ & $\overline{1_{c}[\mu \mathrm{m}]}$ \\
\hline & $\begin{array}{c}\text { Neat } \\
\text { 2W Oxygen Plasma }\end{array}$ & $\begin{array}{l}444 \pm 49 \\
335 \pm 41\end{array}$ & $\begin{array}{l}2687 \pm 631 \\
2950 \pm 731\end{array}$ \\
\hline Epoxy & $\begin{array}{c}\text { 100W Oxygen Plasma } \\
\text { 2W Oxygen Plasma - PECVD } \\
\text { 100W Oxygen Plasma - PECVD }\end{array}$ & $\begin{array}{l}317 \pm 44 \\
355 \pm 30 \\
247 \pm 63\end{array}$ & $\begin{array}{l}1885 \pm 204 \\
2473 \pm 399 \\
1899 \pm 377\end{array}$ \\
\hline Vinylester & $\begin{array}{c}\text { Neat } \\
\text { 2W Oxygen Plasma - PECVD } \\
\text { 100W Oxygen Plasma - PECVD }\end{array}$ & $\begin{array}{l}830 \pm 343 \\
680 \pm 182 \\
460 \pm 116\end{array}$ & $\begin{array}{l}3938 \pm 804 \\
4175 \pm 702 \\
3075 \pm 600\end{array}$ \\
\hline
\end{tabular}

Results also show that, for both thermoset matrices, the best results have been found for the TVS deposition after the $100 \mathrm{~W}$ oxygen pretreatment, indicating a strong enhancement of the yarn/matrix interfacial adhesion. This tendency has been confirmed by micro computed tomography. Using the AVIZO 9.0 software, it was possible to perform a post- mortem 3-D reconstruction of the fracture zone distribution along the flax yarn. Raw images were segmented and the black voids (breaking zone) were identified from the background (the rest of flax yarn) on the basis of the different grey threshold values. This analysis has been performed for both epoxy and vinylester systems with untreated or 100W oxygen - PECVD treated flax yarns (Figure 9). In order to simplify the identification, the dark gray and the green colors have been used for yarn representation, and the blue and red colors have been chosen for damage reconstruction. 

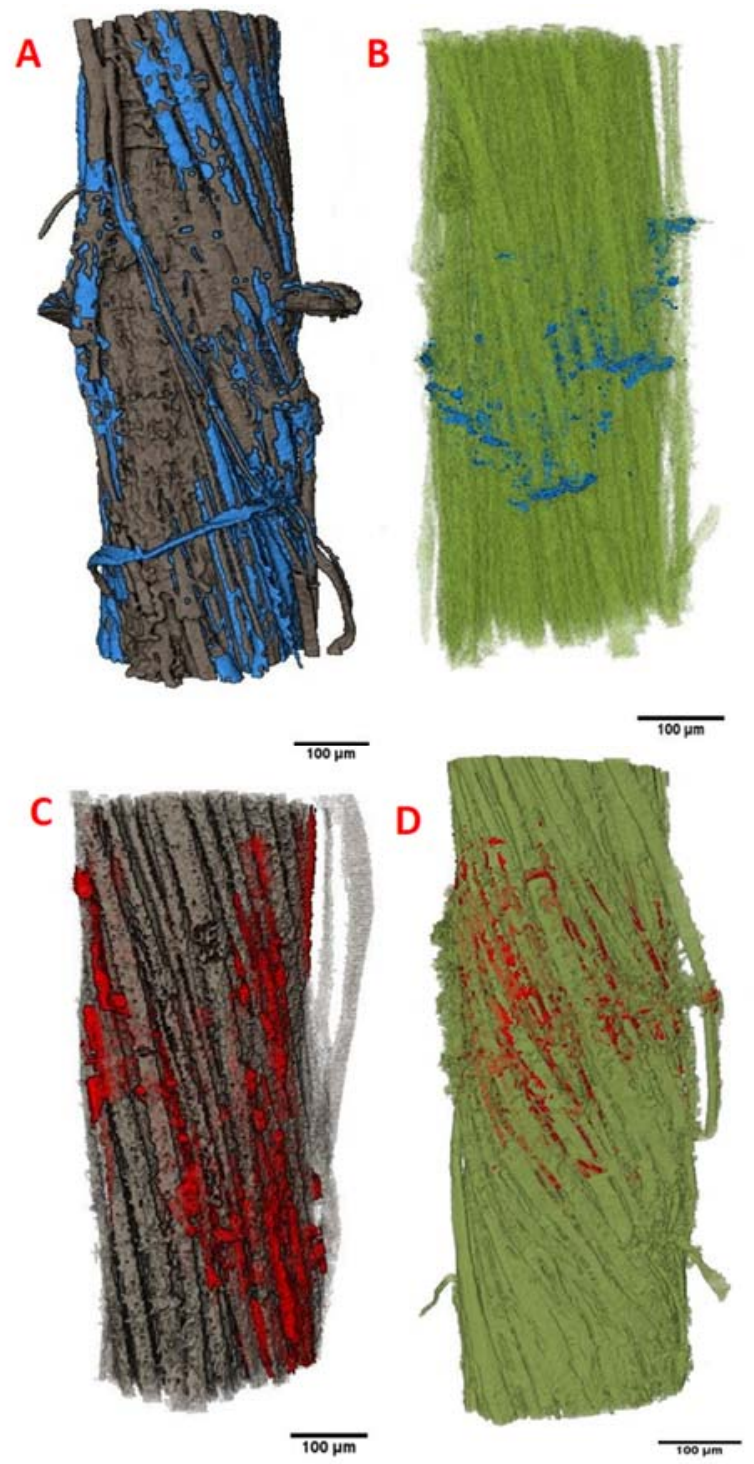

Figure 9 Volumetric reconstruction of the flax yarn and the fracture zone for neat flax/epoxy (A), 100W oxygen - PECVD flax/epoxy (B), neat flax/vinylester (C), 100W oxygen - PECVD flax/vinylester (D) systems.

The 3D reconstructions for both epoxy and vinylester composites highlight the decrease in the debonding length thanks to the pp-TVS film deposition treatment. By comparing the volumetric reconstructions reported in Figure 9-B and Figure 9-D, it is possible to see that the flax yarn/epoxy observations allowed a precise measurement of the yarn/matrix debonding zone. The debonding length has been identified by the black voxels corresponding to voids between the periphery of the 

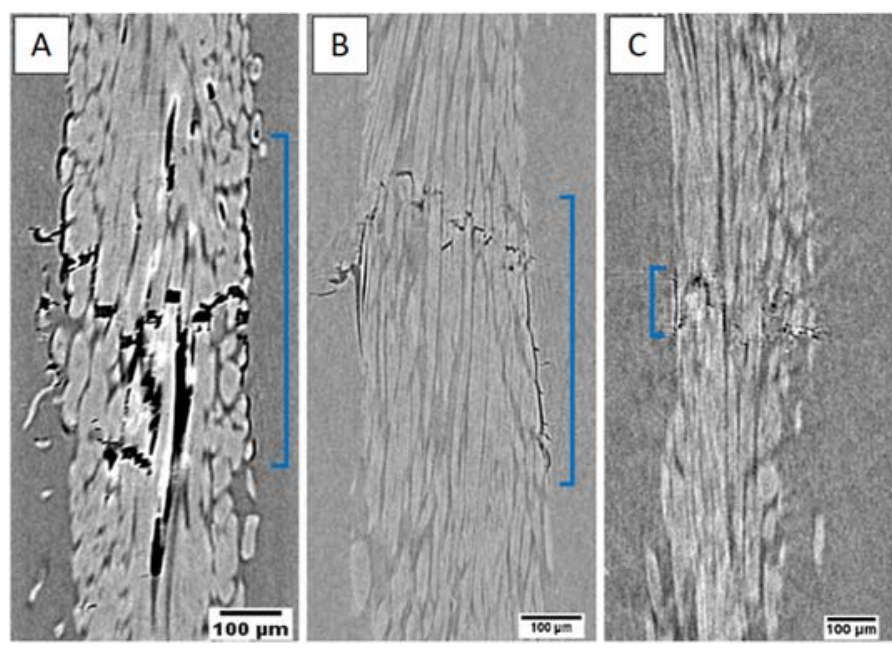

Figure 10 Debonding length observation by micro computed tomography for: neat flax/epoxy (A), 2W oxygen - PECVD flax/epoxy (B) and 100W oxygen - PECVD flax/epoxy (C) samples.
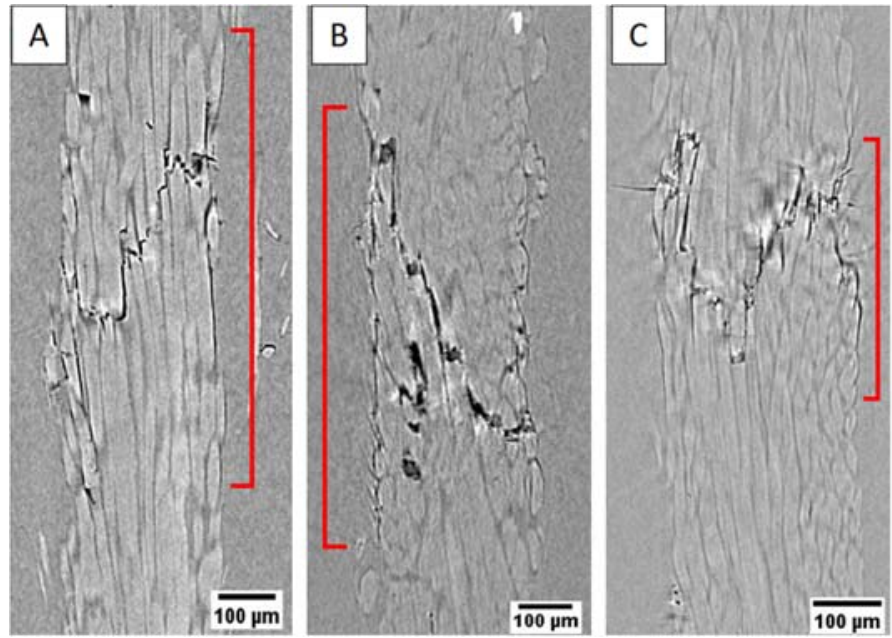

Figure 11 Debonding length observation by micro computed tomography for: neat flax/vinylester (A), 2W oxygen - PECVD flax/vinylester (B) and 100W oxygen - PECVD flax/vinylester (C).

Comparison of debonding lengths confirms that for both thermoset matrices, the polymer deposition on flax yarn after the $100 \mathrm{~W}$ oxygen pre-treatment allows a significant increase of the yarn/matrix interfacial adhesion. Comparison of Figure 10-C and Figure 11-C also confirms that the flax yarn/epoxy monofilament composite shows a better interface quality than the flax/vinylester one.

\section{3 - Calculation of interfacial shear strength}

According to Kelly and Tyson (Kelly and Tyson, 1965), the interfacial shear strength value (IFSS) may be estimated using equation 6 :

$$
I F S S=\frac{\sigma_{f}\left(l_{c}\right) \cdot d}{2 \cdot l_{c}}
$$

where $d$ is the fibre diameter, $l_{c}$ is the critical fragment length and $\sigma_{f}\left(l_{c}\right)$ is the fibre strength at a length equal to the critical filament length. Due to the impossibility to determine directly the 
tensile strength of a single yarn at a length equal to the critical fragment length, an extrapolation of strength at critical length $l_{c}$ has been performed by using the Weibull cumulative distribution function (Guillebaud-Bonnafous et al., 2012; R. Joffe, Andersons, and Wallström, 2005; Roberts Joffe, Andersons, and Wallström, 2003; Zafeiropoulos, 2007). Using the obtained two-parameter

$$
\sigma_{f}\left(l_{c}\right)=\sigma_{f}\left(L_{0}\right)\left(\frac{L_{0}}{l_{c}}\right)^{-\frac{1}{m}}
$$

where $m$ is the shape parameter of Weibull distribution for the tested gauge length $L_{0}$ of $40 \mathrm{~mm}, l_{c}$ is the critical fragment length and $\sigma_{f}\left(L_{0}\right)$ is the strength value at the gauge length. In table 5 are reported all the $\sigma_{f}\left(l_{c}\right)$ values and the IFSS results obtained.

Table 5 Strength values at critical fragment length and IFSS values for the different flax/epoxy and flax/vinylester systems.

\begin{tabular}{cccc}
\hline \hline Matrix & Flax Yarn & $\boldsymbol{\sigma}_{\mathbf{f}}\left(\mathbf{I}_{\mathbf{c}}\right)[\mathbf{M P a}]$ & IFSS [MPa] \\
& Neat & $406 \pm 18$ & $19.3 \pm 3.7$ \\
${ } }$ & 2W Oxygen Plasma & $353 \pm 9$ & $20 \pm 3.8$ \\
& 100W Oxygen Plasma & $363 \pm 11$ & $24.8 \pm 2.8$ \\
& 2W Oxygen Plasma - PECVD & $336 \pm 8$ & $21.2 \pm 1.6$ \\
& 100W Oxygen Plasma - PECVD & $573 \pm 31$ & $41.3 \pm 11.6$ \\
& Neat & $376 \pm 15$ & $13.9 \pm 2.8$ \\
Vinylester & 2W Oxygen Plasma - PECVD & $309 \pm 8$ & $12.4 \pm 2.1$ \\
& 100W Oxygen Plasma - PECVD & $501 \pm 26$ & $23 \pm 6.7$ \\
\hline \hline
\end{tabular}

The oxygen plasma treatment increases the IFSS values and this is particularly true in the case of the $100 \mathrm{~W}$ plasma power. This reflects the results already found by comparing the critical length and the debonding length values. Concerning the PECVD process, from the results reported in table 5 it may be observed that, for both thermoset matrices, a significant increase in the values of IFSS was produced due to the deposition of the polymeric film after the $100 \mathrm{~W}$ oxygen pretreatment. Comparing the IFSS values found for the epoxy and vinylester systems with the $100 \mathrm{~W}$ oxygen-PECVD treated flax yarns, it is possible again to conclude that the flax yarn/epoxy system has a better interface quality than the flax/vinylester one. In order to better understand the interfacial phenomena, a photoelasticity analysis has also been performed. It allowed to carry out a qualitative study of stress state near yarn breaks and at the interface between the yarn and the matrix. This analysis has been performed for the untreated flax yarn/epoxy and the $100 \mathrm{~W}$ oxygenPECVD treated flax yarn/epoxy systems. The vinylester resin used in this work does not exhibit birefringence phenomena, therefore no photoelasticity analysis has been carried out. Figure 12 presents the different isochromatic patterns observed for the two different yarn/epoxy systems at

518 the saturation of the fragmentation process. 


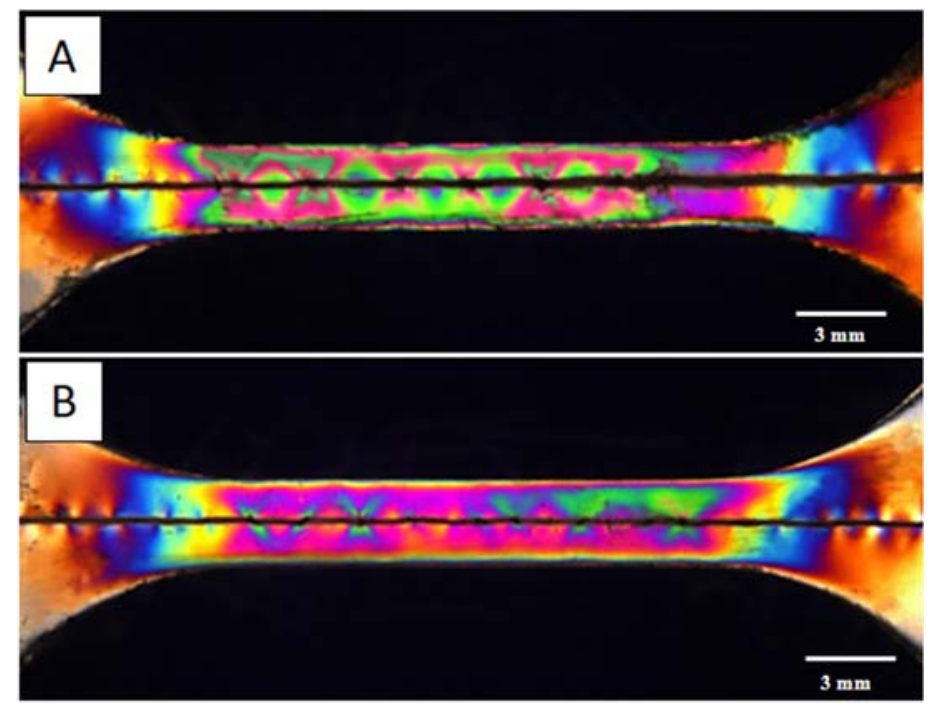

Figure 12 Comparison of experimental photoelastic patterns after yarn fragmentation test for the neat flax yarn/epoxy (A) and the 100W oxygen-PECVD treated flax yarn/epoxy (B) composite specimens.

Around each yarn break, the stress redistribution occurs at $45^{\circ}$ of the tensile loading, showing a cross-shape profile. This type of stress transfer at the interface has been also observed in carbon/epoxy composites (X. Wang et al., 2010). Isochromatic patterns exhibit different fringe orders in Figure 12-A and 12-B. Fringes are more pronounced, with a higher stress level, in the case of the untreated flax yarn than in the case of the PECVD treated one. This dissimilar stress distribution confirms the lower interface quality and the lower IFSS value measured for the

\section{4 - Fractographic analysis}

A post mortem morphological investigation of the fracture surface has been performed using the E-SEM. The fracture surfaces of both single flax yarn samples with epoxy and vinylester resin have been investigated. Figure 13 presents a comparison of the FE-SEM-micrographs showing the fracture surface for the untreated and the different oxygen plasma treated flax/epoxy systems. Observations show that there is a gap in the yarn/matrix interfacial adhesion for both untreated and $2 \mathrm{~W}$ oxygen plasma treated specimens. Indeed, extensive flax fibre debonding can be seen in Figures 13-A and 13-B. On the contrary, for the $100 \mathrm{~W}$ oxygen plasma treated flax/epoxy system, it is possible to notice an increase in the yarn/matrix interfacial adhesion (Figure 13-C). These results are completely in agreement with the critical yarn lengths, the debonding lengths and the IFSS values found. A possible explanation of this behaviour may be found in the plasma oxygen 

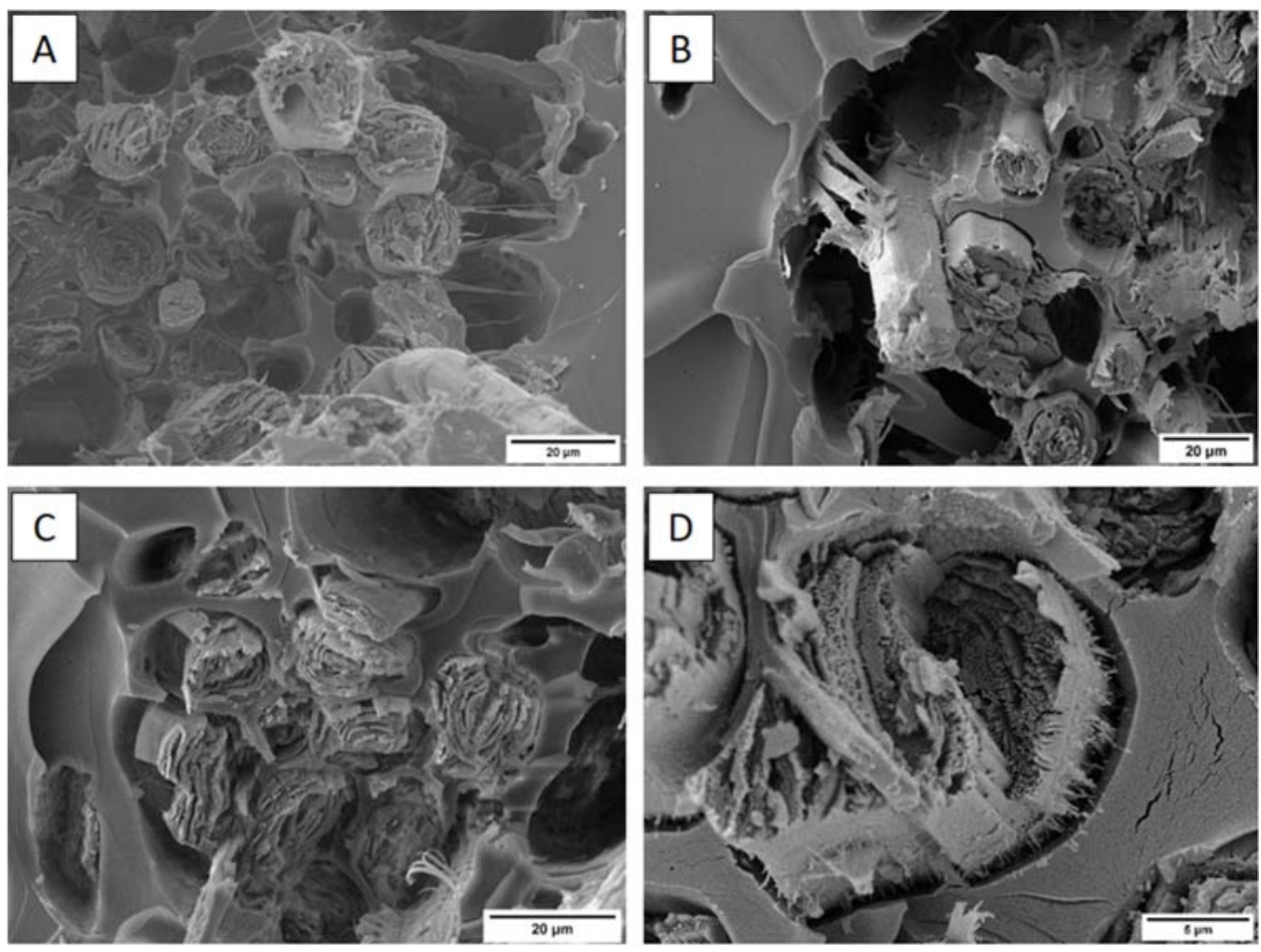

Figure 13 FE-SEM-micrographs showing the fracture surface for the different flax/epoxy single yarn composites: neat flax/epoxy (A), $2 \mathrm{~W}$ oxygen plasma flax/epoxy (B), and 100W oxygen plasma flax/epoxy (C-D).

As discussed previously, oxygen plasma treatment is able to perform a surface ablation of the flax fibres and to enhance their surface roughness (Figure 1). Different studies in literature have analysed the influence of frictional forces on the interfacial adhesion between the synthetic fibres and the polymeric matrices (Beggs et al., 2015; Fu et al., 2019; Gao et al., 2015; Thomason, Yang, and Minty, 2018). These studies showed that the mechanical interlocking action between the fibres surface and the polymer matrix may lead to an increase in the interfacial strength. The increase in mechanical interlocking after plasma treatment can have a great effect on the load transfer between the matrix and the fibres (Bozaci et al., 2013)(De Oliveira et al., 2017)(Kafi, Magniez, and Fox, 2011). For this reason, it is possible to state that the roughness enhancement produced by the oxygen plasma treatment may increase the surface energy of the fibres, increasing the contact area with the polymer matrix and creating static frictional stresses able to enhance the adhesion quality of flax. It is important to highlight also the effect of the oxygen plasma on the chemical composition of flax fibres. During the chemical and ion etching performed by the oxygen plasma treatment, hydrophilic species like hemicelluloses may be degraded. Moreover, the FT-IR analysis has shown that the oxygen plasma treatment performed at $100 \mathrm{~W}$ may promote the formation of oxidized species rich in hydroxyl, carbonyl, carboxyl groups and phenoxy radicals. All these modifications of the surface chemistry of the fibres induced by the oxygen plasma process may have a positive effect on the flax/epoxy adhesion quality. For example, the presence of little resin ligaments between the epoxy matrix and the 100W oxygen treated flax fibres can be observed in Figure 13-D. These little connections may further explain the better adhesion of the $100 \mathrm{~W}$ oxygen treated flax fibres than the $2 \mathrm{~W}$ treated ones. After fragmentation tests, a morphological investigation of the fracture surface has been performed also for the epoxy and vinylester composite samples with PECVD treated flax yarns (Figures 14 and 15, respectively). From an indepth analysis of the different fracture surfaces, it is possible to confirm the higher adhesion of the $100 \mathrm{~W}$ oxygen plasma-PECVD treated flax (Figure 14-D) with respect to the $2 \mathrm{~W}$ oxygen plasmaPECVD treated one (Figure 14-A) with the epoxy resin. The morphological analysis shows that the pp-TVS film, deposited on the flax yarn surface after an oxygen pre-treatment at a plasma power of $2 \mathrm{~W}$, has a good adhesion with epoxy matrix but is completely detached from the surface of the flax fibre (red arrows in Figures 14-B and 14-C). On the opposite, for the 100W oxygen plasma-PECVD treated flax, not only the pp-TVS film is attached to the surface of the epoxy matrix but an improvement in adhesion between the polymeric film and the treated flax fibre surface may be also observed (Figure 14-E). 

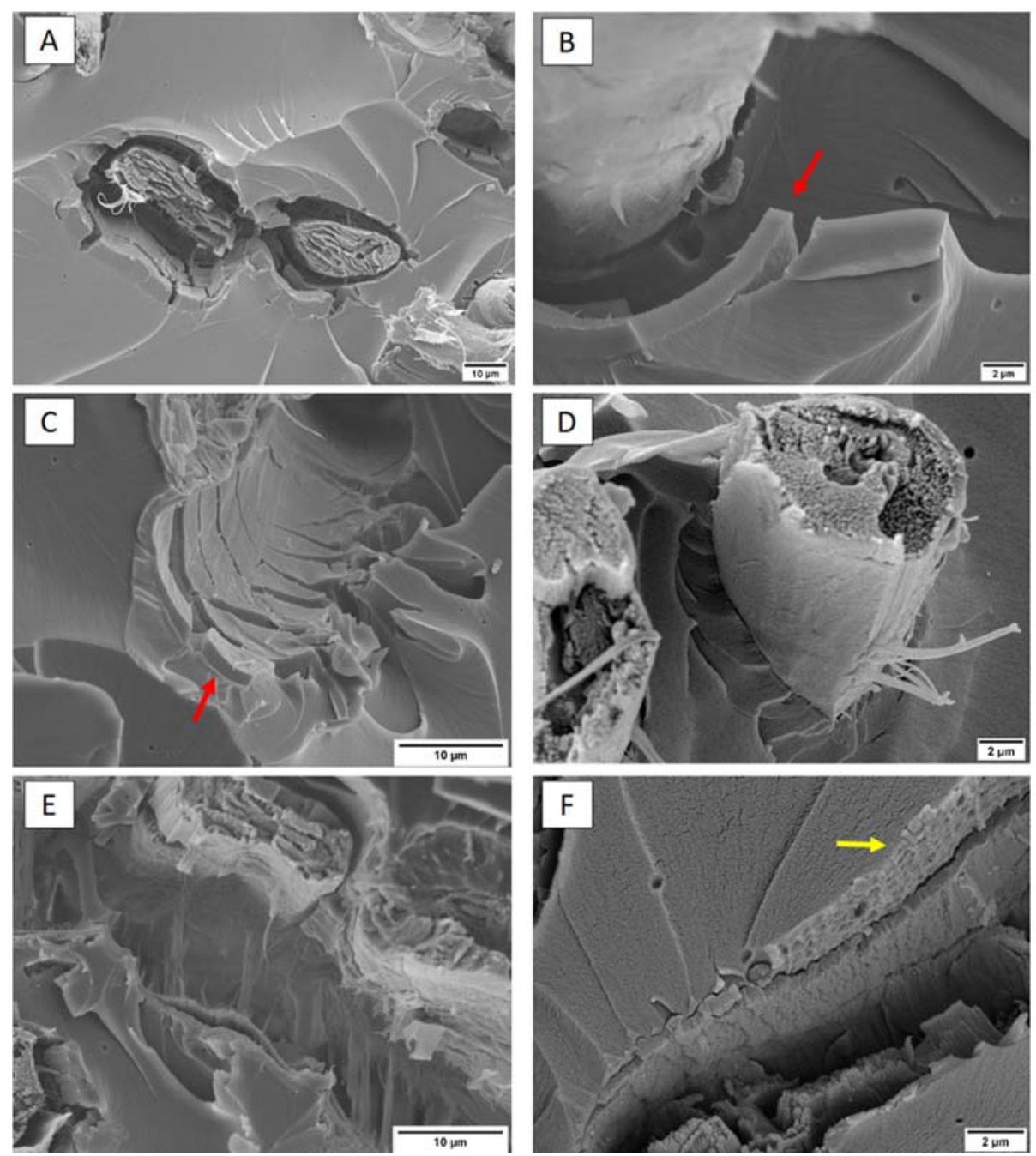

Figure 14 FE-SEM-micrographs showing the fracture surface for the different flax/epoxy single yarn composites: $2 \mathrm{~W}$ oxygen - PECVD flax/epoxy (A-B-C) and 100W oxygen - PECVD flax/epoxy (D-E-F).

From this result, it may be concluded that the oxygen pretreatment plays a fundamental role in the adhesion between the deposited pp-TVS film and the flax surface. Only the 100W oxygen plasma treatment is really able to remove contaminants and improve film adhesion. The morphological analysis allowed to highlight also the effect of the flax yarn roughness on the adhesion with epoxy resin. The higher the plasma oxygen power used, the greater the roughness of the flax fibres. In Figure 14-F, it is possible to see that the epoxy surface that has been directly in contact with the pp-TVS film is highly irregular (yellow arrow) and it may increase the frictional force and the mechanical interlocking between the fibres surface and the polymer matrix. By comparing the fracture surfaces found for the neat flax/vinylester (Figure 15-A) and for the 100W oxygen PECVD flax/vinylester (Figure 15-D) systems, it is possible to see once again that the plasma polymer deposition process is able to produce a strong increase in the fibre/matrix adhesion. Concerning the $2 \mathrm{~W}$ oxygen - PECVD flax/vinylester system, contrary to what has been found for the epoxy resin, the deposited polymer film shows a good adhesion with the fibre surface but, on the contrary, a decohesion towards vinylester resin (red arrows in Figures $15 \mathrm{~B}-\mathrm{C}$ ). This behaviour may explain the lower interfacial adhesion of the $2 \mathrm{~W}$ oxygen - PECVD flax/vinylester composite compared to the 100W oxygen - PECVD flax/vinylester system. 

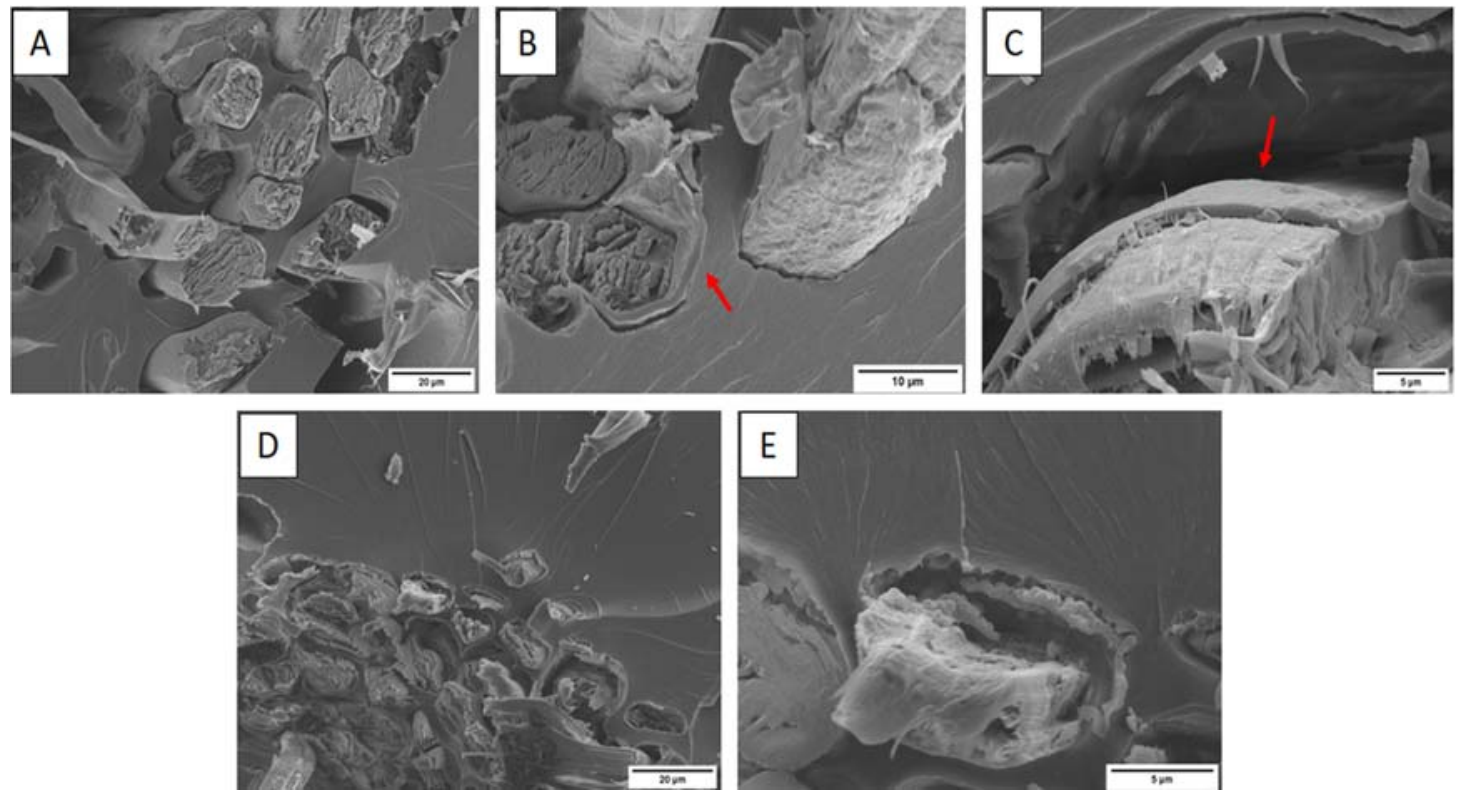

Figure 15 FE-SEM-micrographs showing the fracture surface for the different flax/vinylester single yarn composites: neat flax/vinylester (A), $2 \mathrm{~W}$ oxygen -PECVD flax/vinylester (B-C) and 100W oxygen - PECVD flax/vinylester (D-E).

\section{4. Conclusions}

614 The effect of oxygen and tetravinylsilane plasma treatments on the adhesion of flax yarns with epoxy and vinylester thermoset matrices was evaluated. These low temperature plasma processes represent a more environmentally friendly alternatives to traditional chemical treatments to improve the adhesion of flax yarns with thermoset matrices. The main conclusion of this study is that the plasma polymer deposition is able to produce a significant improvement of the adhesion property of flax yarn with both epoxy and vinylester matrices. Despite a reduction in mechanical properties, a morphological and compositional analysis showed that the oxygen plasma can increase the surface roughness and introduce functional groups in the surface of flax fibres. These effects are important for the enhancement of the fibre/matrix wetting properties and play a key role in promoting the plasma polymer film deposition. In particular, it has been found that the greater the plasma power used during oxygen treatment, the better the adhesion of the polymeric film to the surface of flax fibre. The values of debonding, critical fragment length and IFSS found prove that flax yarns with pp-TVS deposition after the $100 \mathrm{~W}$ plasma oxygen pretreatment exhibit a significant enhancement of the yarn/matrix interfacial adhesion especially for the epoxy matrix. composite materials.

\section{Acknowledgements:}

632 The plasma treatments were supported by the Czech Science Foundation, grant no. 16-09161S. 


\section{References}

Abidi N. and Manike M. (2017), X-ray diffraction and FTIR investigations of cellulose deposition during cotton fiber development. Text. Res. J. 88:719-730.

Abidi N., Cabrales L., and Haigler C. H. (2014), Changes in the cell wall and cellulose content of developing cotton fibers investigated by FTIR spectroscopy. Carbohydr. Polym. 100:9-16.

Amiandamhen S. O., Meincken M., and Tyhoda L. (2018), The effect of chemical treatments of natural fibres on the properties of phosphate-bonded composite products. Wood Sci. Technol. 52:653-675.

Baltazar-y-Jimenez A., Bistritz M., Schulz E., and Bismarck A. (2008), Atmospheric air pressure plasma treatment of lignocellulosic fibres: Impact on mechanical properties and adhesion to cellulose acetate butyrate. Compos. Sci. Technol. 68:215-227.

Beggs K. M., Servinis L., Gengenbach T. R., Huson M. G., Fox B. L., and Henderson L. C. (2015), A systematic study of carbon fi bre surface grafting via in situ diazonium generation for improved interfacial shear strength in epoxy matrix composites. Compos. Sci. Technol. 118:3138.

Bozaci E., Sever K., Sarikanat M., Seki Y., Demir A., and Ozdogan E. (2013), Effects of the atmospheric plasma treatments on surface and mechanical properties of flax fiber and adhesion between fiber - matrix for composite materials. Compos. Part B 45:565-572. http://dx.doi.org/10.1016/j.compositesb.2012.09.042.

Bulut Y. and Aksit A. (2013), A comparative study on chemical treatment of jute fiber : potassium dichromate, potassium permanganate and sodium perborate trihydrate. Cellulose 20:31553164 .

Cech V. et al. (2014), Enhanced interfacial adhesion of glass fibers by tetravinylsilane plasma modification. Compos. Part A 58:84-89. http://dx.doi.org/10.1016/j.compositesa.2013.12.003.

Cech V., Marekb A., Knobc A., Valterb J., Braneckya M., Plihalb P., Vyskocil J. (2019), Continuous Surface Modification of Glass Fibers in a Roll-to-Roll Plasma-Enhanced CVD Reactor for Glass Fiber/Polyester Composites, Compos. Part A: Applied Science and Manufacturing 121: 244-53.

Cech V., Studynka J., Janos F., and Perina V. (2007), Influence of oxygen on the chemical structure of plasma polymer films deposited from a mixture of tetravinylsilane and oxygen gas. Plasma Process. Polym. 4:S776-S780.

Davidson G. (1971), The vibrational spectrum of tetravinylsilane. Spectrochim. Acta Part A Mol. Spectrosc. 27A:1161-1169.

De Almeida Mesquita R. G. et al. (2017), Polyester Composites Reinforced with Corona-Treated Fibers from Pine, Eucalyptus and Sugarcane Bagasse. J. Polym. Environ. 25:800-811.

De Farias J., Cavalcante R., Canabarro B., Viana H., Scholz S., and Simao R. (2017), Surface lignin removal on coir fibers by plasma treatment for improved adhesion in thermoplastic starch composites. Carbohydr. Polym. 165:429-436.

De Oliveira D. M., Cioffi M. O. H., De Carvalho Benini K. C. C., and Voorwald H. J. C. (2017), Effects of plasma treatment on the sorption properties of coconut fibers. Procedia Eng. 200:357364.

De Souza Lima M. M. and Borsali R. (2004), Rodlike Cellulose Microcrystals: Structure, Properties, and Applications. Macromol.ecular Rapid Commun. 25:771-787.

Demirkir C., Colak S., and Ozturk H. (2017), Effects of plasma surface treatment on bending strength and modulus of elasticity of beech and poplar plywood. Maderas. Cienc. y Tecnol. 19:195-202. 
Donaldson L. and Frankland A. (2004), Ultrastructure of iodine treated wood. Holzforschung 58:219-225.

Dong Z., Ding R., Zheng L., Zhang X., and Yu C. (2015), Thermal properties of flax fiber scoured by different methods. Therm. Scienece 19:939-945.

Fiore V., Scalici T., and Valenza A. (2017), Effect of sodium bicarbonate treatment on mechanical properties of flax-reinforced epoxy composite materials. J. Compos. Mater. 52:1061-1072.

Fu J. et al. (2019), Applied Surface Science Enhancing interfacial properties of carbon fi bers reinforced epoxy composites via Layer-by-Layer self assembly GO / SiO 2 multilayers fi lms on carbon fi bers surface. Appl. Surf. Sci. 470:543-554.

Gao X., Jr J. W. G., Jensen R. E., Li W., Haque B. Z. G., and Mcknight S. H. (2015), Effect of fiber surface texture on the mechanical properties of glass fiber reinforced epoxy composite. Compos. Part A 74:10-17.

George J., Sreekala M. S., Thomas S., George J., Sreekala M. S., and Thomas S. (2001), A Review on interface modification and characterization of natural fiber reinforced plastic composites. Polym. Eng. Sci. 41:1471-1485.

Guillebaud-Bonnafous C., Vasconcellos D., Touchard F., and Chocinski-Arnault L. (2012), Experimental and numerical investigation of the interface between epoxy matrix and hemp yarn. Compos. Part A 43:2046-2058. http://dx.doi.org/10.1016/j.compositesa.2012.07.015.

Jamali A. and Evans P. D. (2011), Etching of wood surfaces by glow discharge plasma. Wood Sci. Technol. 45:169-182.

Joffe R., Andersons J. A., and Wallström L. (2003), Strength and adhesion characteristics of elementary flax fibres with different surface treatments. Compos. Part A Appl. Sci. Manuf. 34:603-612.

Joffe R., Andersons J., and Wallström L. (2005), Interfacial shear strength of flax fiber/thermoset polymers estimated by fiber fragmentation tests. J. Mater. Sci. 40:2721-2722.

John M. J. and Anandjiwala R. D. (2008), Recent Developments in Chemical Modification and Characterization of Natural Fiber-Reinforced Composites. Polym. Compos. 29:187-207.

Kafi A. A., Magniez K., and Fox B. L. (2011), A surface-property relationship of atmospheric plasma treated jute composites. Compos. Sci. Technol. 71:1692-1698.

Kalia S., Kaith B. S., and Kaur I. (2009), Pretreatments of Natural Fibers and their Application as Reinforcing Material in Polymer Composites - A Review. Polym. Eng. Sci. 49:1253-1272.

Ke G., Yu W., Xu W., Cui W., and Shen X. (2007), Effects of corona discharge treatment on the surface. J. Mater. Process. Technol. 7:125-129.

Kelly A. and Tyson W. R. (1965), Tensile properties of fibre-reinforced metals: Copper/Tungsten and Copper/Molybdenum. J. Mech. Phys. Solids 13:329-350.

Kiruthika A. V (2017), A review on physico-mechanical properties of bast fi bre reinforced polymer composites. J. Build. Eng. 9:91-99.

Koronis G., Silva A., and Fontul M. (2013), Green composites: A review of adequate materials for automotive applications. Compos. Part B 44:120-127.

Li X., Tabil L. G., and Panigrahi S. (2007), Chemical Treatments of Natural Fiber for Use in Natural Fiber-Reinforced Composites: A Review. J. Polym. Environ. 15:25-33.

Liu M. et al. (2016), Effect of pectin and hemicellulose removal from hemp fibres on the mechanical properties of unidirectional hemp / epoxy composites. Compos. Part A 90:724-735.

Liu M. et al. (2017), Oxidation of lignin in hemp fibres by laccase: Effects on mechanical properties of hemp fibres and unidirectional fibre / epoxy composites. Compos. Part A 95:377-387. 
Lucintel (2015), Growth Opportunities in the Global Natural Fiber Composites Market, Lucintel Insights that Matter, 1-170.

Mazian B., Bergeret A., Benezet J., and Malhautier L. (2018), Influence of field retting duration on the biochemical, microstructural, thermal and mechanical properties of hemp fibres harvested at the beginning of flowering. Ind. Crop. Prod. 116:170-181.

Mohanty A. K., Misra M., and Drazal L. T. (2001), Surface modifications of natural fibers and performance of the resulting biocomposites: An overview. Compos. Interfaces 8:313-343.

Molina S. (2016), Modification of Natural Fibers Using Physical Technologies and Their Applications for Composites., in Lignocellulosic Fibers and Wood Handbook: Renewable Materials for Today's Environment, N. Belgacem and A. Pizzi, Eds. 2016.

Mukhopadhyay S. and Fangueiro R. (2009), Physical Modification of Natural Fibers and Thermoplastic Films for Composites - A Review. J. Thermoplast. Compos. Mater. 22:135-162.

Ng Y. R., Shahid S. N. A. M., and Nordin N. I. A. A. (2018), The effect of alkali treatment on tensile properties of coir/polypropylene biocomposite., in The Wood and Biofiber International Conference (WOBIC 2017), 1-7.

Ohsawa T., Nakayama A., Miwa M., and Hasegawa A. (1978), Temperature dependence of critical fiber length for glass fiber - reinforced thermosetting resins. J. Appl. Polym. Sci. 22:3203-3212.

Ragoubi M. et al. (2012), Effect of corona discharge treatment on mechanical and thermal properties of composites based on miscanthus fibres and polylactic acid or polypropylene matrix. Compos. Part A 43:675-685.

Reddy K. O., Maheswari C. U., Reddy K. R., Shukla M., Muzenda E., and Rajulu A. V. (2015), Effect of Chemical Treatment and Fiber Loading on Mechanical Properties of Borassus (Toddy Palm) Fiber/Epoxy Composites. Int. J. Polym. Anal. Charact. 20:612-626.

Sarasini F., Tirillò J., and Seghini M. C. (2018), Influence of thermal conditioning on tensile behaviour of single basalt fibres. Compos. Part B 132:77-86.

Scalici T., Fiore V., and Valenza A. (2016), Effect of plasma treatment on the properties of Arundo Donax L. leaf fi bres and its bio-based epoxy composites: A preliminary study. Compos. Part B 94:167-175.

Seghini M. C., Touchard F., Sarasini F., Chocinski-arnault L., Mellier D., and Tirillò J. (2018), Interfacial adhesion assessment in flax/epoxy and in flax/vinylester composites by single yarn fragmentation test : Correlation with micro-CT analysis. Compos. Part A 113:66-75.

Shanmugasundaram N., Rajendran I., and Ramkumar T. (2018), Characterization of untreated and alkali treated new cellulosic fi ber from an Areca palm leaf stalk as potential reinforcement in polymer composites. Carbohydr. Polym. 195:566-575.

Sun D. and Stylios G. K. (2006), Fabric surface properties affected by low temperature plasma treatment. Mater. Process. Technol. 173:172-177.

Thomason J. L., Yang L., and Minty R. F. (2018), Are silanes the primary driver of interface strength in glass fibre composites?: exploring the relationship of the chemical and physical parameters which control composite interfacial strength., in ECCM18 - 18th European Conference on Composite Materials Athens, Greece, 24-28th June 2018, 1-8.

Titok V., Leontiev V., Yurenkova S., Nikitinskaya T., Barannikova T., and Khotyleva L. (2010), Infrared Spectroscopy of Fiber Flax. J. Nat. Fibers 7:61-69.

Velde K. Van De and Baetens E. (2001), Thermal and Mechanical Properties of Flax Fibres as Potential Composite Reinforcement. Macromol. Mater. Eng. 286:342-349. 
Wang B., Panigrahi S., Tabil L., Crerar W., Sokansanj S., and Braun L. (2003), Modification of flax fibers by chemical treatment., in CSAE/SCGR 2003 Meeting Montréal, Québec July 6 - 9, 2003, 1-15.

Wang X., Zhang B., Du S., Wu Y., and Sun X. (2010), Numerical simulation of the fiber fragmentation process in single-fiber composites. Mater. Des. 31:2464-2470. http://dx.doi.org/10.1016/j.matdes.2009.11.050.

Warner S.B., Uhlmann D.R., and Peebles Jr L.H. (1975), Ion etching of amorphous and semicrystalline fibres. J. Mater. Process. Technol. 10:758-764.

Yachmenev V. G., Blanchard E. J., and Lambert A. H. (1998), Use of Ultrasonic Energy in the Enzymatic Treatment of Cotton. Mater. Interfaces 37:3919-3923.

Yasuda H., Matsuzawa Y., (2005), Economical Advantages of Low-Pressure Plasma Polymerization Coating. Plasma Process. Polym. 2:507-512.

Zafeiropoulos N. E. (2007), On the use of single fibre composites testing to characterise. Compos. Interfaces 14:807-820. 